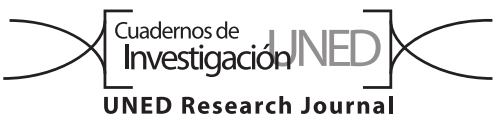

\title{
Tamaño corporal de cuatro especies de mariposas del género Heliconius (Lepidoptera: Nymphalidae) producidas en zoocriaderos del Caribe de Costa Rica
}

\author{
Olivier Castro Morales, ${ }^{1}$ Arnoldo Gadea Rivas ${ }^{1}$ \& Mónica Madrigal Valverde ${ }^{1}$ \\ 1. Instituto Tecnológico de Costa Rica, Campus Tecnológico Local San Carlos, Escuela de Agronomía, 223-21001 Alajuela, San Carlos, Costa Rica; \\ ocastro@itcr.ac.cr; (iD) https://orcid.org/0000-0003-3817-9459, \\ agadea@itcr.ac.cr; (iD) https://orcid.org/0000-003-4063-9856, \\ madrigalvalverdem@gmail.com; (iD https://orcid.org/0000-0002-4688-0627
}

Recibido 26-VI-2019 • Corregido 23-VII-2019 • Aceptado 16-VIII-2019

DOI: https://doi.org/10.22458/urj.v11i3.2699

\begin{abstract}
Body size of four species of butterflies of the genus Heliconius (Lepidoptera: Nymphalidae) produced in Costa Rica Caribbean butterfly farms". Introduction: The production of butterflies is an important socioeconomic activity and has become an option to generate income for rural microenterprises. Objective: To see how farming affects the body size of Heliconius butteflies in Costa Rican farms. Methods: We measured adults and pupae of Heliconius cydno, $H$. erato, $H$. hecale and $H$. melpomene in 10 butterfly farms, in the Huetar Atlantic Region of Costa Rica. Results: In some farms, adult Heliconius erato and $H$. hecale are heavier $(p \leq 0.05)$, and have a longer abdomen and larger forewings. The weight did not differ among farms, and the sex ratio is close to 1:1. Conclusion: All farms sampled produce butterflies of fit for commercialization.
\end{abstract}

Key words: Lepidoptera, biometrics, Heliconius, butterfly farms, breeding of butterflies.
RESUMEN: Introducción: La producción de mariposas es una actividad socioeconómica importante y se ha convertido en una opción para generar ingresos en las microempresas rurales. Objetivo: Observar como los zoocriaderos afectan el tamaño corporal de mariposas Heliconius, producidas en zoocriaderos de Costa Rica. Métodos: Medimos adultos y pupas de Heliconius cydno, $\mathrm{H}$. erato, $\mathrm{H}$. hecale y $\mathrm{H}$. melpomene de 10 zoocriaderos de mariposas, ubicados en la zona Huetar Atlántica de Costa Rica. Resultados: En algunos zoocriaderos las mariposas adultas de Heliconius erato y $\mathrm{H}$. hecale son significativamente más pesadas ( $\mathrm{p} \leq$ $0,05)$, poseen un abdomen más largo y el ala anterior más grande. El peso no difiere entre zoocriaderos, las relaciones de sexos son cercanas a 1:1. Conclusiones: Todos los zoocriaderos muestreados producen mariposas con calidad para su comercialización.

Palabras clave: Lepidóptera, biometría, Heliconius, zoocriadero, cría de mariposas.
El manejo en cautiverio es una estrategia para la conservación de especies y para que esta actividad sea exitosa "requiere integrar principios de manejo de alta calidad, cuidado sanitario, manejo genético y consideraciones especiales para poblaciones pequeñas" (RangelMendoza \& Weber, 2015, p. 500).

Los lepidópteros a diferencia de otros grupos taxonómicos son insectos que permiten el manejo en zoocriaderos o viveros, siendo estos una alternativa potencial para el uso y conservación de la especie como de sus ecosistemas, ya que se trata de un recurso renovable (Montero, 2007), pero para poder hacer este manejo se requiere de una mayor investigación y sistematización de experiencias en la producción de las mariposas en sus diferentes estadios.
El manejo sobre las plantas hospederas y nectaríferas, para la producción de mariposas, puede influir en la productividad (Sánchez, 2004, Claro \& Ruiz, 2010). En los insectos fitófagos existe la especialización a través de las generaciones en la preferencia de una o unas pocas plantas huésped (Friberg \& Wiklund, 2009; Nylin, Nygren, Soderlind, \& Stefanescu 2009).

El éxito de Costa Rica en la producción de mariposas se debe a factores tales como la ubicación geográfica, políticas de gobierno, sistemas de telecomunicaciones, territorios naturales protegidos y el $5 \%$ de la biodiversidad mundial entre sus fronteras (Brinckerhoff, 1999).

El sector productivo de mariposas de Costa Rica es un gremio organizado alrededor de pocas empresas 
exportadoras, tales como: El Bosque Nuevo, Spirogyra, Kasiri S.A., Paraíso de Mariposas S.A. y Suministros Entomológicos Costarricenses S. A. (Valverde \& Barrantes, 2008).

La zoocría de mariposas tropicales, en Costa Rica ha generado una creciente demanda de estos lepidópteros, principalmente por el aumento del turismo, la exhibición de mariposas vivas en sitios donde se recrean ecosistemas naturales y a la educación ambiental con mariposas (CAF, SGCAN, \& UNCTAD, 2005; PROCOMER, 2015).

El objetivo de la presente investigación es realizar una caracterización biométrica de cuatro especies de mariposas del género Heliconius (Nimphalidae), producidas en diez zoocriaderos de mariposas, en el Caribe de Costa Rica.

\section{MATERIALES Y MÉTODOS}

Los zoocriaderos de mariposas incluidos en el estudio se encuentran debidamente inscritos ante Sistema Nacional de Áreas Conservación (SINAC), cumpliendo con sus obligaciones incluidas en el plan de manejo, indicadas en la Ley de Manejo y Conservación de la Vida Silvestre (7317) y su reglamento (La Gaceta, 2017).

El estudio se realizó desde enero del 2016 a diciembre del 2017, se seleccionaron 10 zoocriaderos de mariposas ubicados en el Caribe de Costa Rica, distribuidos de la siguiente forma: dos en el Distrito de Germania del Cantón de Siquirres, dos en el Distrito de Guácimo del Cantón de Guácimo, dos en el Distrito de Río Jiménez de Guácimo, tres en el distrito de Guápiles en el Cantón de Pococí, todos estos de la Provincia de Limón y uno en la Provincia de Heredia, Distrito Horquetas del Cantón Sarapiquí. Abarcando tres áreas de conservación: Área de Conservación la Amistad Caribe; subregión SiquirresMatina, Área de Conservación Tortuguero; Sub-región Pococí y Área de Conservación Cordillera Volcánica Central, subregión Norte. Estas áreas de conservación se caracterizan por poseer una alta concentración de zoocriaderos de mariposas y por ende una alta productividad de lepidópteros.

Para determinar las características biológicas de las mariposas, seleccionamos cuatro especies de mariposas: Heliconius cydno, Heliconius erato, Heliconius melpomene y Heliconius hecale, todas de la familia Nymphalidae, subfamilia Heliconiinae, se utilizaron estas mariposas, debido a que los 10 zoocriaderos seleccionados para el presente estudio tenían la capacidad de producir estas especies durante el período de la investigación de manera continua.
Debido a la producción estacional de las mariposas Heliconius en los zoocriaderos, antes de realizar la visita para la recolección de las mariposas adultas y las pupas, se coordinó con antelación la visita con cada uno de los propietarios de los zoocriaderos.

El método de recolección de las mariposas adultas fue realizado utilizando una red entomológica con $50 \mathrm{~cm}$ de diámetro. En cada zoocriadero se recogieron de forma sistemática diez mariposas adultas por especie, evitando hacer capturas cerca de las plantas hospederas para reducir la probabilidad de que la mariposa capturada fuera una hembra que estuviese ovipositando.

Las variables evaluadas en los adultos fueron: el peso, la longitud del abdomen, tamaño del ala anterior y tamaño del ala posterior.

En cada mariposa adulta se midió el largo del abdomen, el tamaño del ala derecha anterior y posterior, para hacer las medidas se utilizó un calibrador $( \pm 0,01 \mathrm{~mm})$, adicionalmente se pesó cada adulto en una romana electrónica $( \pm 0,01 \mathrm{~g})$ y se registró el sexo de cada mariposa, esto se realizó por observación directa, ya que las hembras de las mariposas Heliconius exponen sus genitales cuando se manipulan del abdomen y el macho presenta unas valvas, las cuales no son expuestas.

Para determinar la calidad biométrica de las pupas de mariposas producidas en cada uno de los zoocriaderos, fueron seleccionadas diez pupas de forma aleatoria de todas las producidas en el zoocriadero para el día del muestreo, se identificó la especie de Heliconius a la que pertenecía cada pupa.

Fueron evaluado el tamaño y peso de la pupa. El tamaño fue medido determinando la longitud desde el cremáster hasta la cabeza, sin incluir las proyecciones cefálicas, la medida fue tomada utilizando un calibrador electrónico $( \pm 0,01 \mathrm{~mm})$, para registrar el peso individual de la pupa se utilizó una romana electrónica $( \pm 0,01 \mathrm{~g})$.

Para el análisis estadístico de la información de las características físicas de los individuos, categorizados por zoocriadero y por especie. Fue empleado un modelo lineal generalizado mediante el proceso mixto (GLMix) del software estadístico InfoStat-Statistical/P. Se observó la homocedasticidad y normalidad, se realizaron correcciones para heterocedasticidad en las varianzas evaluadas, la comparación de medias fue realizada por medio de la prueba de DGC con un nivel de significancia de $p<0,05$. La prueba Chi-cuadrado $\left(x^{2}\right)$ se utilizó para verificar las diferencias en la proporción de sexos entre las cuatro especies de mariposas estudiadas.

Adicionalmente, se realizó una correlación lineal de Pearson para asociar las variables de peso, largo del 
abdomen, tamaño del ala anterior y posterior con el sexo de la mariposa, entre las especies.

Ética, conflicto de intereses y declaración de financiamiento: Los autores declaran haber cumplido con todos los requisitos éticos y legales pertinentes, tanto durante el estudio como en el manuscrito; que no hay conflictos de interés de ningún tipo, y que todas las fuentes financieras se detallan plena y claramente en la sección de agradecimientos. Asimismo, están de acuerdo con la versión editada final del documento. El respectivo documento legal firmado se encuentra en los archivos de la revista.

\section{RESULTADOS}

No hubo diferencia $(p \leq 0,05)$ para las variables de peso en los machos y las hembras adultas entre las cuatro especies de mariposas de Heliconius estudiadas. Al analizar esta variable el peso entre los zoocriaderos, se encontró diferencias en Heliconius erato (ANDEVA: $p<0,0001 ; g l=5$; $n=55$ ), donde el zoocriadero del productor nueve fue el que presentó tamaños menores en comparación con los otros zoocriaderos. Para la especie Heliconius hecale
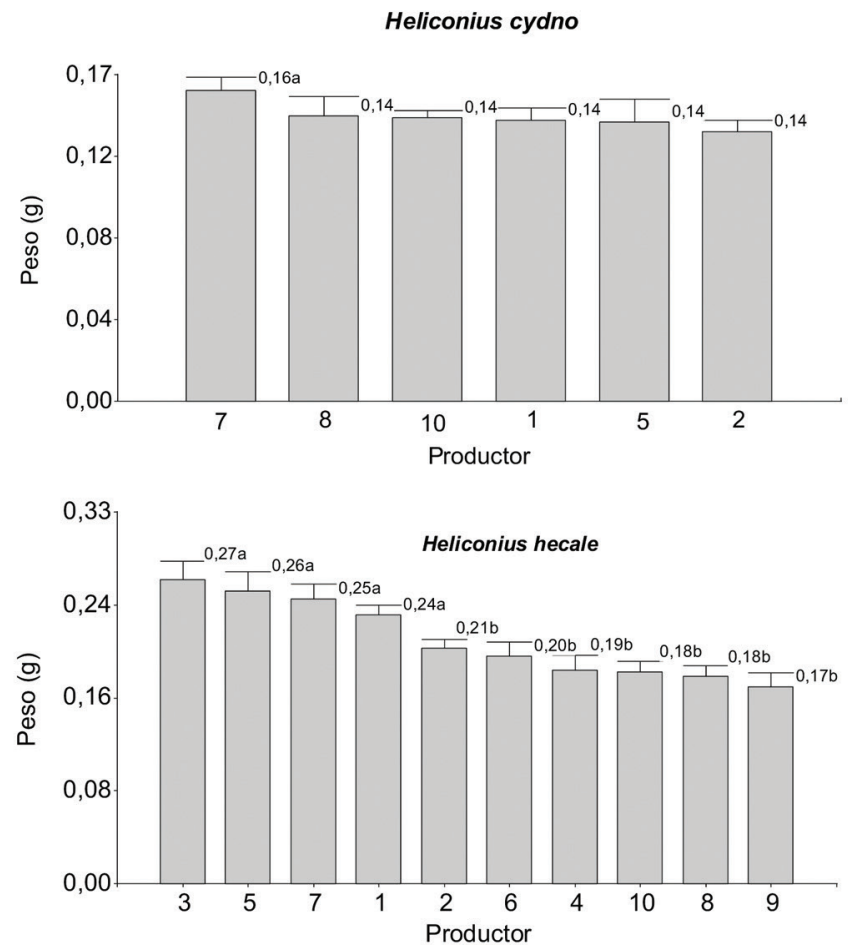

hubo diferencia en el peso de los adultos (ANDEVA: $p<$ 0,$0001 ; g l=9 ; n=100$ ), donde los productores $1,3,5$ y 7 , presentaron pesos mayores que el resto de los productores y para Heliconius cydno y $\mathrm{H}$. Melpomene, no se encontró ninguna diferencia en el peso de los adultos entre zoocriaderos (Fig. 1).

El tamaño del abdomen de las mariposas adultas de Heliconius erato fueron diferentes entre productores (ANDEVA: $\mathrm{p}=0,0082 ; \mathrm{gl}=5 ; \mathrm{n}=55$ ), y lo mismo ocurrió para Heliconius hecale (ANDEVA: $p=0,0001 ; g l=9 ; n=100$ ). Mientras, Heliconius cydno y H. Melpomene, no presentaron ninguna diferencia en el tamaño del abdomen entre productores (Fig. 2).

El tamaño del ala anterior derecha de la mariposa adultas de Heliconius hecale presentó diferencias en el tamaño (ANDEVA: $p<0,0001 ; g l=9 ; n=100$ ), igual para el ala posterior derecha (ANDEVA: $p<0,0008 ; g l=9 ; n=100$ ), Heliconius erato solo presentó diferencias en el tamaño del ala posterior derecha entre productores (ANDEVA: $p=$ 0,0010; $\mathrm{gl}=5 ; \mathrm{n}=55$ ). Las mariposas adultas de Heliconius cydno y $\mathrm{H}$. Erato no presentan diferencias en el tamaño de las alas entre zoocriaderos (Fig. 3).

El peso de las pupas de las mariposas analizadas fue diferente entre productores. Las pupas de Heliconius
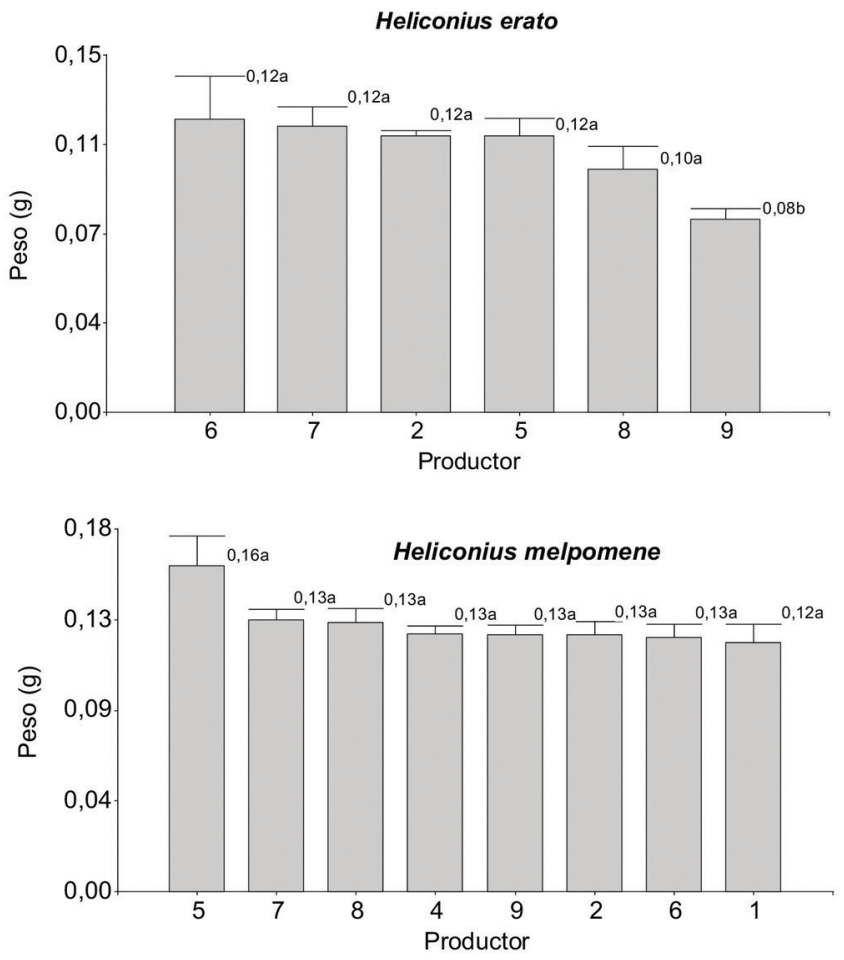

Fig. 1. Peso promedio en gramos $( \pm 0,01 \mathrm{~g})$ y error estándar (E.E) de cuatro especies de mariposas adultas del género Heliconius, en zoocriaderos del Caribe de Costa Rica, según productor (2016-2017). *Medias con letra común dentro de la columna no son significativamente diferentes $(P \geq 0,05)$, según la prueba de DGC. 

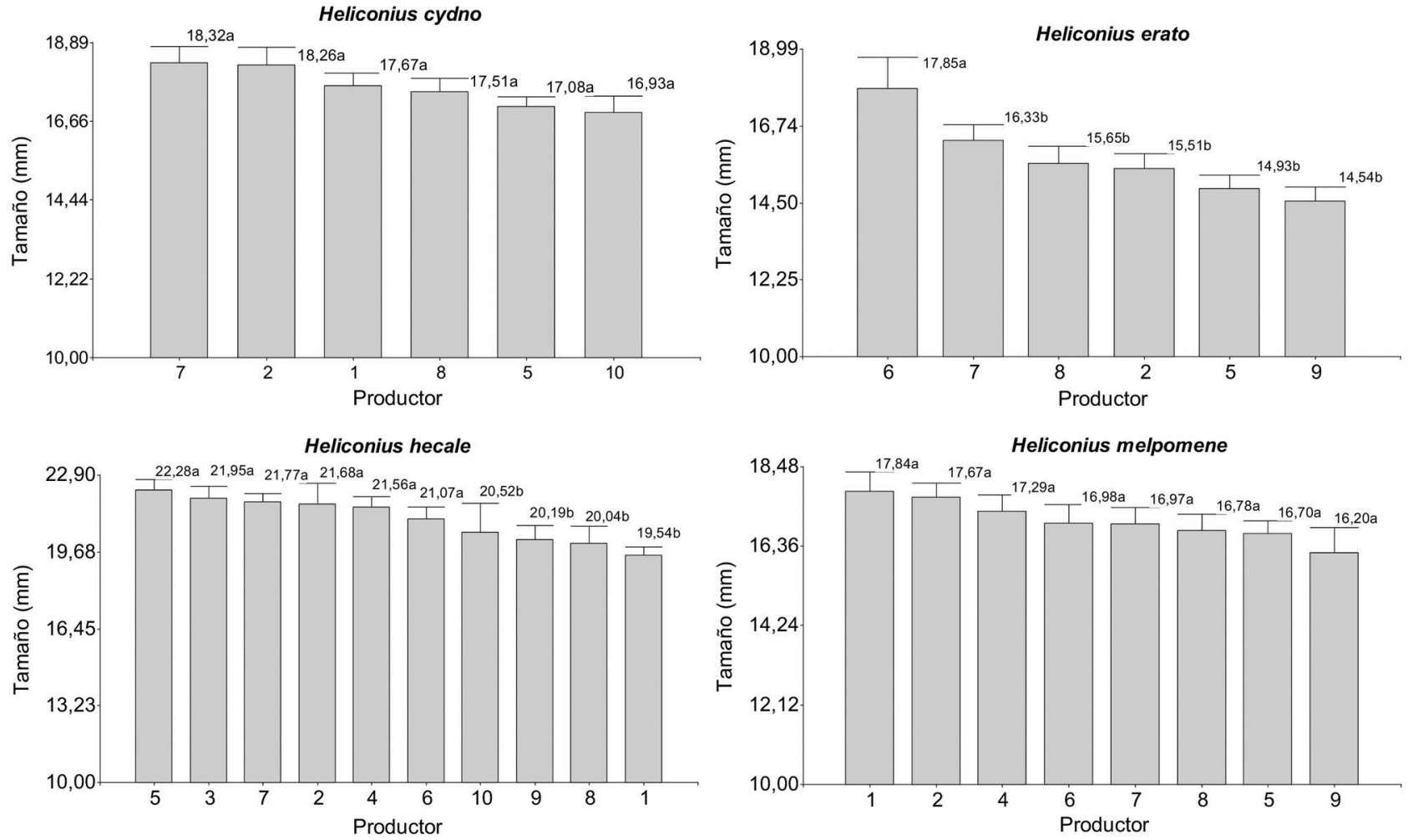

Fig. 2. Tamaño del abdomen $( \pm 0,01 \mathrm{~mm})$ y error estándar $(E . E)$ de cuatro especies de mariposas adultas del género Heliconius, en zoocriaderos del Caribe de Costa Rica, según productor (2016-2017). *Medias con letra común dentro de la columna no son significativamente diferentes $(p \geq 0,05)$, según la prueba de DGC.
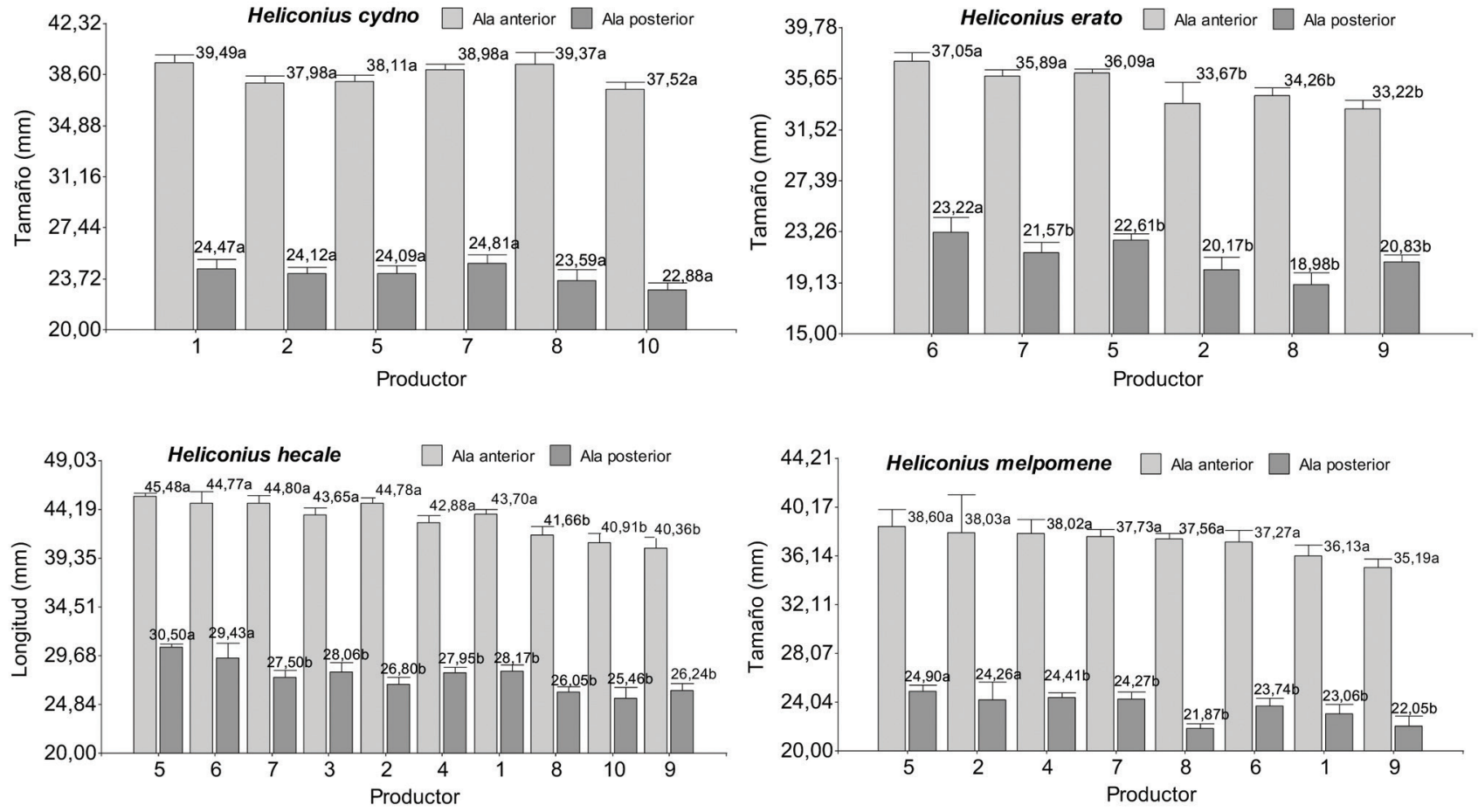

Fig. 3. Tamaño del ala anterior y posterior $( \pm 0,01 \mathrm{~mm})$ y error estándar (E.E) de cuatro especies de mariposas adultas del género Heliconius, en zoocriaderos del Caribe de Costa Rica, según productor (2016-2017). *Medias con letra común dentro de la columna no son significativamente diferentes $(P \geq 0,05)$, según la prueba de DGC. 
erato presentaron un peso promedio mayor en zoocriaderos de los productores uno y siete, siendo semejantes entre sí; pero diferentes en los zoocriaderos de los otros productores uno y ocho (ANDEVA: $p=0,0069 ; g \mathrm{l}=3$; $\mathrm{n}=40$ ). El grupo de Heliconius cydno presentó diferencias en el peso de las pupas entre productores (ANDEVA: $p$ $<0,0001 ; \mathrm{gl}=3 ; \mathrm{n}=38$ ), donde el productor siete tuvo las pupas menos pesadas. Heliconius hecale presenta diferencias en el peso entre productores, las pupas de mayor peso para esta especie fue en productores uno, cinco y seis, entre ellos no hay diferencias, pero si difieren entre el resto de los productores (ANDEVA: $p<0,0001 ; g l=8$; $\mathrm{n}=86$ ). Heliconius melpomene también presentó diferencias en el peso de la pupa entre productores (ANDEVA:
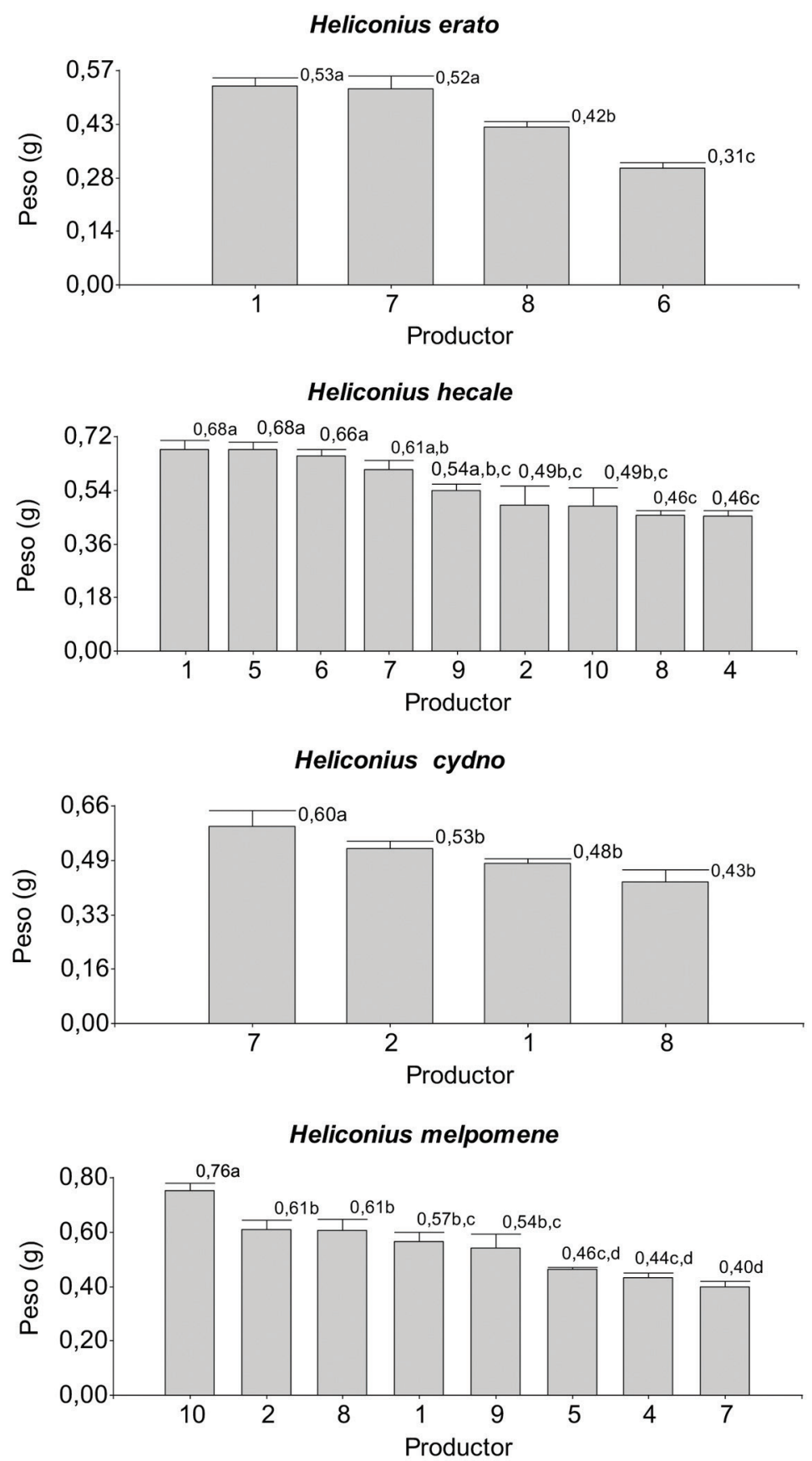

Fig. 4. Peso de las pupas $( \pm 0,01 \mathrm{~mm})$ y error estándar (E.E) de cuatro especies de mariposas adultas del género Heliconius, en zoocriaderos del Caribe de Costa Rica, según productor (2016-2017). *Medias con letra común dentro de la columna no son significativamente diferentes $(P \geq 0,05)$, según la prueba de DGC. 

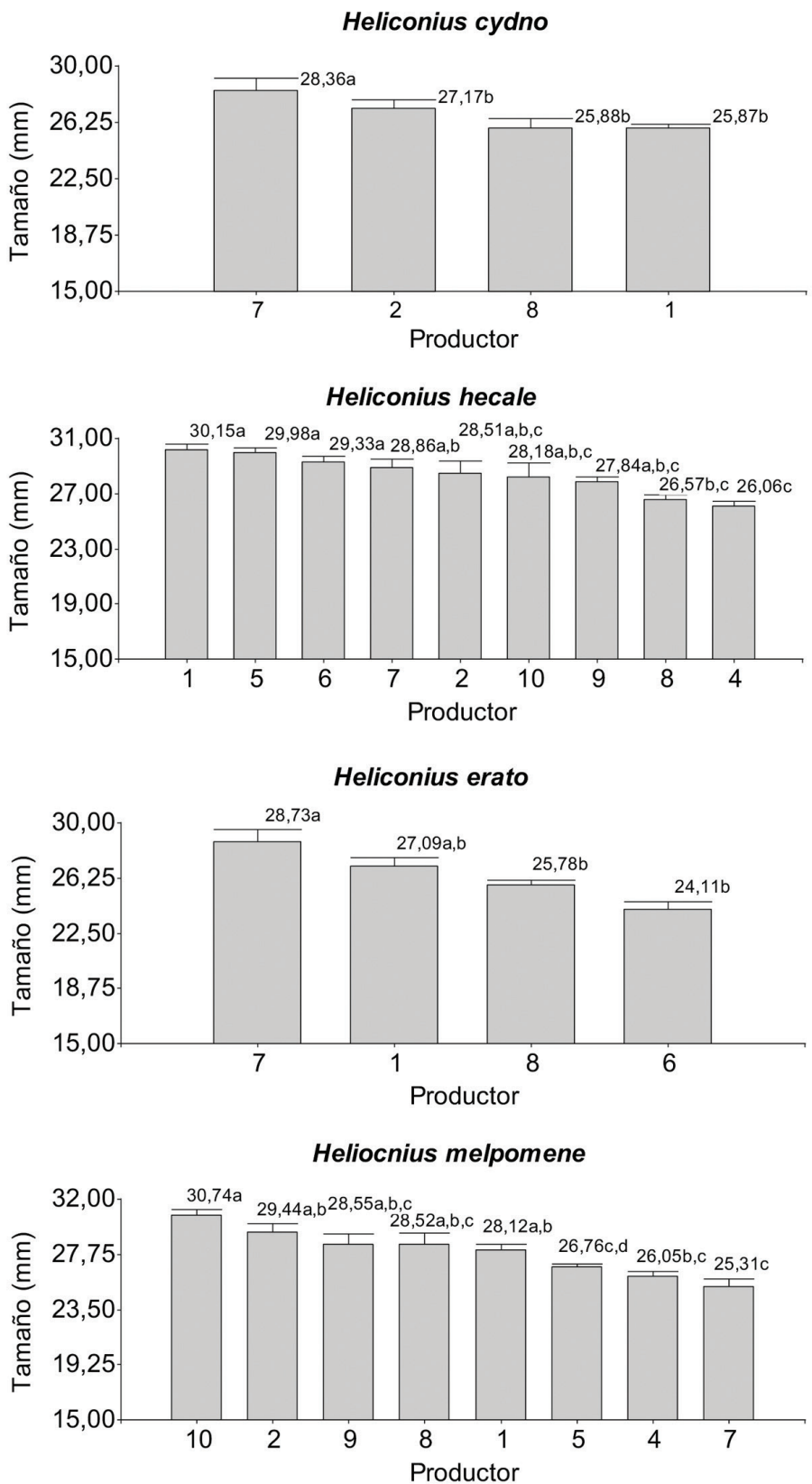

Fig. 5. Tamaño de las pupas $( \pm 0,01 \mathrm{~mm})$ y error estándar $(E . E)$ de cuatro especies de mariposas del género Heliconius, en zoocriaderos del Caribe de Costa Rica, según productor (2016-2017). *Medias con letra común dentro de la columna no son significativamente diferentes $(P \geq 0,05)$, según la prueba de DGC.

$\mathrm{p}<0,0001 ; \mathrm{gl}=7 ; \mathrm{n}=80$ ), el productor diez presentó un mayor peso que el resto de los productores (Fig. 4).

Las pupas de las mariposas analizadas presentaron diferencias en el tamaño según sea el productor. Las pupas de Heliconius cydno presentan un tamaño mayor en el zoocriadero siete (ANDEVA: $p=0,0183 ; g l=3 ; n=40$ ). Las pupas de Heliconius erato presentó un tamaño en los zoocriaderos del productor seis y siete las pupas presentaron un mayor tamaño (ANDEVA: $p<0,0001 ; \mathrm{gl}=3 ; \mathrm{n}=38$ ). Las pupas de Heliconius hecale presentan un tamaño mayor en los zoocriaderos de los productores uno, cinco y seis, pero entre ellos no hay diferencias significativas, sí difieren entre los productores ocho y cuatro. Sin embargo, entre estos últimos no hay diferencias y el que presenta menor promedio en el tamaño de la pupa es en el zoocriadero del productor cuatro, el cual es diferente a todos los demás (ANDEVA: $\mathrm{p}<0,0001 ; \mathrm{gl}=8 ; \mathrm{n}=86$ ) (Fig. 5). 


\section{CUADRO 1}

Análisis de correlación de cuatro variables para cuatro especies de mariposas del género Heliconius, producidas en zoocriaderos del Caribe de Costa Rica.

\begin{tabular}{lccccccc}
\multicolumn{1}{c}{ Especie } & $\mathrm{n}$ & $\begin{array}{c}\text { Peso*Tamaño } \\
\text { abdomen } \mathrm{P} \leq 0,05\end{array}$ & $\mathrm{r}^{2}$ & $\begin{array}{c}\text { Peso*Tamaño } \\
\text { ala anterior } \mathrm{P} \leq 0,05\end{array}$ & $\mathrm{r}^{2}$ & $\begin{array}{c}\text { Tamaño abdomen*Tamaño } \\
\text { ala anterior } \mathrm{P} \leq 0,05\end{array}$ & $\begin{array}{c}\mathrm{r}^{2} \\
\text { Heliconius Cydno }\end{array}$ \\
Heliconius erato & 60 & 0,2578 & 0,1484 & 0,0554 & 0,2487 & 0,0515 \\
Heliconius hecale & 100 & $\mathbf{0 , 0 0 4 9}$ & 0,3742 & $\mathbf{0 , 0 1 4 3}$ & 0,3286 & $\mathbf{0 , 0 0 0 1}$ \\
Heliconius melpomene & 80 & $\mathbf{0 , 0 1 4 3}$ & 0,244 & $<\mathbf{0 , 0 0 0 1}$ & 0,5457 & $\mathbf{0 , 0 0 0 1}$ & 0,4951 \\
\hline
\end{tabular}

** números en negrilla indican coeficientes de correlación significativos $(p<0,05)$.

\section{CUADRO 2}

Análisis de correlación del tamaño de Abdomen y el ala anterior para hembras y machos de cuatro especies de mariposas del género Heliconius, producidas en zoocriaderos del Caribe de Costa Rica.

\begin{tabular}{|c|c|c|c|c|}
\hline Variable & Especie & Sexo & $P \leq 0,05$ & $r^{2}$ \\
\hline \multirow[t]{8}{*}{ tamaño abdomen*tamaño ala anterior } & Heliconius cydno & Hembra & 0,0150 & 0,4806 \\
\hline & & Macho & 0,7633 & $-0,0528$ \\
\hline & Heliconius erato & Hembra & 0,0413 & 0,3748 \\
\hline & & Macho & 0,0021 & 0,5846 \\
\hline & Heliconius hecale & Hembra & 0,0260 & 0,3212 \\
\hline & & Macho & 0,0083 & 0,3622 \\
\hline & Heliconius melpomene & Hembra & 0,0086 & 0,4050 \\
\hline & & Macho & 0,0730 & 0,2904 \\
\hline
\end{tabular}

* números en negrilla indican coeficientes de correlación significativos $(p<0,05)$.

El tamaño promedio de las pupas de Heliconius melpomene fue diferente entre productores, donde las pupas del productor diez, presentaron un mayor tamaño que el resto de productores y el tamaño de las pupas en los productores uno, dos y seis fueron similares entre ellos pero diferentes al resto, mientras que las pupas que presentar en promedio menor tamaño son las de los productores cuatro y cinco, estos son similares estadísticamente entre sí, pero diferentes al resto de los productores, (ANDEVA: $p<0,0001 ; g l=7 ; n=80$ ) (Fig. 5).

Respecto a la correlación entre las variables analizadas Heliconius cydno, no presentó ninguna correlación, pero en Heliconios erato y Heliconius hecale se encontró correlaciones entre las variables analizadas y en la especie Heliconius melpomene, no hubo correlación entre la variable peso y el tamaño del abonen (Cuadro 1).

La correlación lineal entre el tamaño del abdomen con el tamaño del ala anterior en los machos y hembras se cumple en casi todos los casos, para las cuatro especies de mariposas estudiadas. (Cuadro 2). También se observó una correlación positiva entre el peso y el tamaño del ala anterior para las hembras $(P=<0,0001 ; n=48$; $\mathrm{r} 2=0,6522)$ y los machos $(\mathrm{P}=0,0002 ; \mathrm{n}=52 ; \mathrm{r} 2=0,4902) \mathrm{de}$ Heliconius hecale y para las hembras $(P=0,0047 ; n=41$; $r 2=0,4331)$ y machos $(P=0,0011 ; n=39 ; r 2=0,5047)$ de Heliconius melpomene.

En los zoocriaderos de mariposas del Caribe de Costa Rica, la relación entre machos y hembras son cercanas a 0,5:0,5 y no son estadísticamente diferentes para Heliconius cydno, $H$. erato, $H$. hecale y $H$. melpomene (Cuadro 3).

\section{DISCUSIÓN}

El buen estado de los mariposarios es esencial para el éxito de la producción de mariposas, debido a que esto evita el escape de mariposas que se crían en cautiverio y que estas se apareen con mariposas silvestres (Montero, 2007). En todos los zoocriaderos estudiados presentan poca luminosidad, las mallas o el sarán estaban saturados de líquenes, lo que puede reducir la ventilación interna del zoocriadero, los sistemas de ingreso y egreso de algunos fuse deficiente o poco funcional, lo que eventualmente se debería analizar ya que esto 
CUADRO 3

Relación entre hembra y macho para cuatro especies de mariposas producidas en los zoocriaderos del Caribe de Costa Rica.

\begin{tabular}{lcccc}
\multicolumn{1}{c}{ Especie } & Sexo & Relación & $\mathrm{n}$ & $\mathrm{X}^{2}$ \\
Heliconius cydno & Hembra & 0,42 & 25 & \multirow{2}{*}{0,4856} \\
Heliconius erato & Macho & 0,58 & 35 & \\
\multirow{3}{*}{ Heliconius hecale } & Hembra & 0,55 & 30 & \multirow{2}{*}{0,4175} \\
& Macho & 0,45 & 25 & \\
Heliconius melpomene & Hembra & 0,48 & 48 & \multirow{2}{*}{0,0697} \\
& Macho & 0,52 & 52 & \\
& Hembra & 0,51 & 42 & 0,1404 \\
\hline
\end{tabular}

puede bajar la calidad y cantidad de pupas producidas en los zoocriaderos.

La empresa de Suministros Entomológicos Costarricenses S.A. inicio en la producción y exportación de mariposas en Costa Rica en el año 1985 (Chacón, 2003), quien hasta la fecha sigue siendo la empresa que más comercializa pupas en Costa Rica, abasteciéndose de proveedores de todo el país. Por este motivo es importante realizar estudios y monitores de biometrías de mariposas, producidas en zoocriaderos para garantizar un suministro con altos estándares de calidad para la exportación, ya que el precio depende de la calidad (Centro de Inteligencia sobre Mercados Sostenibles, 2006).

De Vries (1987), reporta para Heliconius cydno el tamaño del ala anterior entre $38-43 \mathrm{~mm}$ y Montero (2007) reporta para esta misma especie que el ala anterior del macho mide entre $33-45 \mathrm{~mm}$ y la hembra entre $36-43 \mathrm{~mm}$, coincidiendo con lo encontrado en nuestro estudio, para los zoocriaderos del Caribe de Costa Rica.

El tamaño del ala anterior de Heliconius erato, son semejantes a los reportados en la literaria por De Vries (1987) y Rios, et. al. (2013) y mayores a los reportados por Montero (2007), con esta información se puede decir que las mariposas Heliconius z|erato en ambos sexos, están recibiendo una adecuada nutrición y manejo en los mariposarios del Caribe de Costa Rica, ya que son similares a los evaluados en estado silvestre.

Los machos de la mariposa Heliconius hecale tienen un tamaño mayor del ala anterior que los reportados por De Vries (1987) y por Montero (2007), mientras que, para los otros rangos estudiados en ambos sexos, se encuentra dentro de los reportados por los autores antes mencionados,

Las mariposas Heliconius melpomene, presento un tamaño promedio del ala anterior dentro de los parámetros reportados por De Vries (1987).
En general el tamaño del ala anterior de las cuatro especies de Heliconius estudiadas comparadas con lo reportado en la literatura, estas mariposas se encuentran dentro de esos rangos de tamaño, por lo que se puede concluir que las mariposas criadas en zoocriaderos en el Caribe de Costa Rica se encentra recibido las condiciones necesarias para su buen desarrollo físico.

A pesar de que no encontramos diferencias significativas entre la relación de hembras y machos en las diferentes especies de Heliconius estudiadas, se ha observado que por lo general los machos de las mariposas se presentan en mayor número, que las hembras y muchas hipótesis han sido formuladas para explicar esta desviación en la proporción de sexos de 1:1 encontradas en mariposas criadas en 25 laboratorios (Ehrlich, 1984). Una de las explicaciones del fenómeno es que, las hembras son menos susceptibles a la captura, otra es que las hembras migran más por el néctar, lo que las hace estar más dispersas y también se cree que esta condición de más machos que hembras puede cambiar de generaciones en generaciones con las condiciones ecológicas y algunas excepciones se dan por el control genético (Freitas, 1993).

Las diferencias estadísticas encontradas entre especies, de mariposas en los zoocriaderos del Caribe de Costa Rica posiblemente están influenciadas por las condiciones del suelo, los tratamientos agronómicos recibidos en el manejo de las plantaciones y composición nutritiva de las plantas hospedera y la distribución del alimento dentro del zoocriadero (De Andrade \& Freitas, 2005). Uno de los factores que más influencian la estructura de una población es la distribución y abundancia de los recursos nutricionales (Ehrlich, 1984).

El éxito de la zoocría de mariposa depende de las fuentes de alimento que dispongan los adultos y las larvas, ya que estas sustancias intervienen en su longevidad, supervivencia, fecundidad y actividades de vuelo 
(Montero, 2007), por esta razón se recomienda recurrir a los análisis de suelo y foliares para correlacionarlos con características biométricas de los individuos de mariposas en sus diferentes estadios.

A través de las visitar realizadas a los zoocriaderos de mariposas del Caribe de Costa Rica, se observó que estos son manejados de forma artesanal, los productores no poseen capacitación formal, y no realizan prácticas agrícolas sistemáticas. A pesar de esto, las condiciones de las mariposas producidas en sus zoocriaderos son buenas como producto final y están entre los parámetros normales de crecimiento, lo que las hace aptas para la exportación.

Los productores de mariposas del Caribe no están organizados en asociaciones, cooperativas o cualquier otro gremio, debido a esto ellos están expuestos a los bajos precios de comercialización de las mariposas en sus diferentes estadios, por las empresas dedicadas a la comercialización de lepidópteros y otros insectos en Costa Rica.

La mayoría de los productores de mariposa en Costa Rica están ubicados en el Caribe (Montero, 2007), pero no existen registros científicos que permita comparar la calidad de las mariposas que los productores ofrecen a los mercados internacionales. Por lo que recomendamos a las autoridades ambientales hacer este tipo de investigación, para todas las especies de mariposas producidas en zoocriaderos de todo el país.

\section{AGRADECIMIENTOS}

Este trabajo fue parte del proyecto de Investigación "Caracterización de sistemas productivos de pupas de mariposas (Lepidoptera: Nymphalidae) para la exportación en la Región Huetar Atlántica de Costa Rica" código 2151, inscrito en la Vicerrectoría de Investigación y Extensión del Instituto Tecnológico de Costa Rica. Agradecemos a los productores de mariposas: Geovany Gutiérrez C., Ana Yuri Brenes J, Marvin Elizondo M., Patricia Mejia V., Eduardo Lange G., Adrián Coto C. Ligia Gutiérrez C., Marisol Gutiérrez C., Roberto Gutiérrez F. quienes amablemente facilitaron el ingreso a sus sistemas productivos, cooperando con la información y material requerido para llevar a cabo esta investigación, a la Vicerrectoría de Investigación y Extensión del ITCR, por facilitar tiempo para los investigadores y soporte económico para ejecutar este trabajo y a José Manuel Cordero (Holy), quien siempre nos acompañó como guía de la zona.

\section{REFERENCIAS}

Brinckerhoff, J. (1999). La cría de mariposas: Una industria agrícola maravillosa en papel. Conferencia 165. Memoria XI Congreso Agronómico Nacional y de recursos naturales. (pp. 521-527). San José, Costa Rica.

CAF (Corporación Andina de Fomento)., Secretaría General De La Comunidad Andina (SGCAN)., Conferencia de Las Naciones Unidas Sobre Comercio y Desarrollo (UNCTAD). (2005). Biocomercio en la subregión Andina: Oportunidades para el desarrollo. Recuperado de: http:// scioteca.caf.com/handle/123456789/661.

Centro de Inteligencia sobre Mercados Sostenibles. (2006). Análisis del potencial de captura, montaje y venta de mariposas exóticas para mejorar el ingreso de los habitantes de los bosques en América Central. Recuperado de: http:// www.incae.edu/es/clacds/publicaciones/pdf/cen565. pdf

Chacón, A. I. (2003). El Impacto de las granjas de mariposas en los ecosistemas y el quehacer de los costarricenses. Caterpillar Biodiversity Whorkshop, (p.19-20). Santa Rosa, Guanacaste.

Claro, R. A., \& Ruiz, N. (2010). Aceptación de una dieta artificial por orugas de la mariposa Battus polydamas (Lepidoptera: Papilionidae). Acta Biológica Colombiana, 15 (1), 47-62.

De Andrade, R. B., \& Freitas, A. V. L. (2005). Population Biology of two species of Heliconius (Nymphalidae: Heliconiinae) in a semi-deciduous forest in southeastern Brazil. Journal of the Lepidopterists Society, 59(4), 223-228

DeVries, P. J. (1987). The Butterflies of Costa Rica, and Their Natural History. Nueva Jersey, Estados Unidos: Princeton.

Ehrlich, P. R. (1984). The structure and dynamics of butterfly populations. En: Vane-Wright, R. I. \& Ackery, P. R. (Eds.). The biology of butterflies. (pp. 25-40). London: Academic Press.

Freitas, A. V. L. (1993). Biology and population dynamics of Placidula euryanassa, a relict Ithomiine butterfly (Nymphalidae: Ithomiinae). Journal of the Lepidopterists Society, 47(1), 87-105.

Friberg, M., \& Wiklund, C. (2009). Host plant preference and performance of the sibling species of butterflies Leptidea sinapis and Leptidea reali: a test of the trade-off hypothesis for food specialization. Oecología, 159 (1), 127137. DOI: 10.1007/s00442-008-1206-8.

La Gaceta (2017, 29 de agosto). Reglamento a la Ley de Conservación de la Vida Silvestre № 7317. La Gaceta, № 194, San José, Costa Rica: Imprenta Nacional.

Montero, R. J. (2007). Manual para el manejo de mariposarios. Heredia, Costa Rica: INBio.

Nylin, S., Nygren, G. H., Soderlind, L., \& Stefanescu, C. (2009). Geographical variation in host plant utilization in the comma butterfly: the roles of time constraints and 
plant phenology. Evolutionary Ecology, 23(1), 807-825. DOI:10.1007/s10682-008-9274-0.

PROCOMER (Promotora de Comercio Exterior de Costa Rica) (2015). Exportaciones costarricenses de la partida 0511.99.90.20 (Huevos y pupas para la cría de mariposas). Información suministrada vía correo electrónico del 26 de marzo del 2015.

Rangel-Mendoza, J. A., \& Weber, M. (2015). Evaluación del estado físico de la tortuga blanca, Dermatemys mawii, bajo condiciones de cautiverio en Tabasco, México. Agrociencia 49, 499-511.

Rios, G. G., Gonçalves, S. M., Aparecida, R. P. D. Rodrigues, S. J., Peixoto, A. P., Miranda, N. C., Lange, D., \& Stefani, V.
(2013). Population structure of two species of Heliconius kluk, 1780 butterflies (Lepidoptera: Nymphalidae). Bioscience Journal, 29 (5), 1350-1360.

Sánchez, L. R. (2004). Protocolo de cría para dos especies de mariposas, Assia monuste y Leptophobia aripa (Lepidoptera: Pieridae) bajo condiciones controladas en el Municipio de la Mesa, Cundicamarca (Trabajo de grado), Pontificia Universidad Javeriana, Bogotá, Colombia.

Valverde, K., \& Barrantes, M. (2008). Manual de exportación de pupas de mariposa a Estados Unidos. (Trabajo Comunitario Universitario). Universidad de Costa Rica, San José, Costa Rica. 